Citation: Çavuş, F, Beken, M , Özcanlı, Y . "An Sem Study of Pp/Pet Blends". Journal of Engineering Technology and Applied Sciences 1 (3) $2016: 127-131$

\title{
AN SEM STUDY OF PP/PET BLENDS
}

\author{
Fatma Kosovalı Çavuş $^{a^{*}}$, Murat Beken ${ }^{b}$, Yeşim Özcanlı ${ }^{\mathrm{c}}$ \\ $a^{*}$ Department of Electronics Techonologies, Vocational School Halic,University Istanbul, Turkey \\ fatmacavus@halic.edu.tr(corresponding author) \\ ${ }^{c}$ Department of Electric-Electronic Engineering, Faculty of Engineering, Beykent University Istanbul, \\ Turkey \\ muratbeken@beykent.edu.tr
}

${ }^{c}$ Department of Physics, Faculty of Arts\&Science, Yildiz Technic University Istanbul, Turkey

lenger@yildiz.edu.tr

\begin{abstract}
In this study, the morphology and spectroscopic properties of polypropylene (PP)/ polyethyleneterephthalate (PET) blends were investigated by PET addition to improve the mechanical properties of polypropylene. For the morphological characteristics of the PP + PET blends, SEM analysis for the spectroscopic properties and FT-IR analysis were performed. As the percentage of the mixture increases in the SEM images, there is a gap between the PET and the structure depending on the addition ratio. In the PP $+40 \%$ PET mixture, the transition from droplet form to fibril form appears clearly in the structure.
\end{abstract}

Keywords: Polymer, PP/PET blends, morphology.

\section{Introduction}

Because polymeric materials are highly non-linear systems, the experimental and theoretical calculations of physical and chemical properties polymer materials also their modeling are not easy. Most of the polymer materials used in polymeric applications are homopolymers. However, complex applications require more complex materials. But simple homopolymer systems can not provide the necessary conditions for complex applications.Furthermore, the development of new polymer systems to create the right materials is a long and very expensive process.

Polymer blends, an alternative method for the development of new polymer systems, are the physical mixing of two or more polymers to obtain new materials. These blends are prepared for the purpose of reducing the price of expensive polymers, increasing the processability of heat-sensitive polymers, or improving the impact resistance of homopolymers etc. [1]. 
In this study, scanning electron microscope (SEM) analysis and Fourier transform infrared spectroscopy (FT-IR) spectroscopic properties of polypropylene (PP)/ polyethyleneterephthalate (PET) mixtures obtained by making a polyethyleneterephthalate (PET) additive as a suitable alternative to glassy fill additive to improve this property of polypropylene (PP) have been investigated.

Both PP and PET are examples of semicrystalline polymers. The semi-crystalline polymers are partially amorphous and partially crystalline. Therefore, they show two thermal behaviors: glass transition temperature ( $\mathrm{Tg}$ ) and melting temperature (Te). PP is in the polyolefin family and is often used in applications such as packaging, textiles, recyclable containers, laboratory materials, loudspeakers and car parts [2]. In addition, both academic and commercial circles have received great interest and the production of PP has shown a great increase, and PP shows a brilliant future as a building material in the next century [3].

PET one of the most produced plastics in the world, is a linear polyester. PET is used in synthetic fiber, coating, packaging and plastic construction. The PET polymer is highly resistant to abrasion and alkalines with a high modulus of elasticity [4].

Although PP and PET are widely used alone, there are very few studies on polyolefin / polyester blends in the market and in the literature [5]. The interest in polyolefins and PET blends is increasing due to the improvement of the mechanical properties due to poor partnership. PP in polymer blends is widely preferred due to its good processability and low cost. The melting and glass transition temperatures of PET and PP are very different. For this reason, PET strengthens the tensile strength of PP at high temperatures [5]. If the morphology of the PP / PET blends can be optimized, these blends are a potential application area since these properties are critical as packaging material.The final product, which is the result of mixing these two materials while preserving their superior properties, exhibits better barrier, workability and durability compared to their lean state [2].

In addition, studies with PP / PET blends are limited in the literature. Literature studies have been undertaken to improve the properties of mixtures with different compatibilizers and additives in order to eliminate the incompatibility due to the polarity and chemical differences of the PP / PET blends

The effect of PP / PET blends with PP-G-AA compatibilizer was investigated by Si et al. And as a result, the size of PET droplets was noticeably reduced with harmonizing effect, and microfibrils were observed to be formed by the combination of PET droplets They showed mechanical improvement due to the improvement of the interfacial interface, and DSC results showed that PET could support heterogeneous nucleation agent as well, which is beneficial to the crystallization of PP [1].

\section{Experimental method}

In this study, PP / PET mixtures was used with maleic anhydride functionalized polypropylene (PP-MA) as compatibilizer and the amount of PP decreased accordingly as the amount of PET in the blends increased $(0,10 \%, 20 \%, 30 \%, 40 \%, 50 \%, 60 \%)$. SEM analyzes were performed with a LEOL SEM (SEM, JEOL Ltd., JSM-5910LV) device at X50 magnification after Au/Pd coating. The FTIR analyzes for the characterization of the PP / PET mixtures were carried out with Brumer brand LUMOS model FT-IR between 4000-650 cm -1. 
The mixtures were compared to the characteristic peaks of pure materials to see the interactions in PP / PET blends in the form of film containing 10\%, 20\%, 30\%, 40\%, 50\% and $60 \%$ PET

\section{Results and discussions}

\subsection{SEM analysis}

In the images obtained from the SEM analyzes, the main building forms PP. In the PP $+10 \%$ PET blends, the structure appears to be irregularly distinct. Within the structure, droplet-shaped PET polymer is present at different diameters and in heterogeneous form, and structure is showed that not a distinctive adhesion

In Figure 1, PET particles in the PP $+20 \%$ PET mixture are converted into droplets, and the structure is heterogeneous and voids appear in the structure. $\mathrm{PP}+40 \%$ PET sample has a more homogeneous structure. In this example, it can be assumed that the voids have decreased and the PET droplets have settled into the voids. It is also observed that fibrillar structures began to form slowly in this sample. In PP $+40 \%$ PET, the fibrils expand and appear more clearly. In the case of PP $+50 \%$ PET, the homogeneity starts to deteriorate again and we can say that the voids start to form again and that the PET particles completely cover the main structure

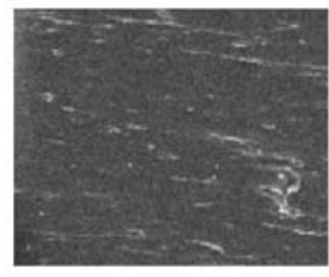

a) PP

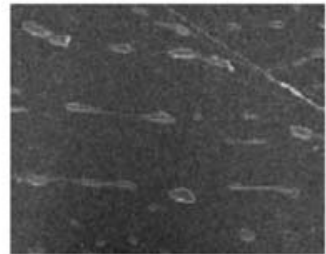

b) $\mathrm{PP}+10 \% \mathrm{PET}$

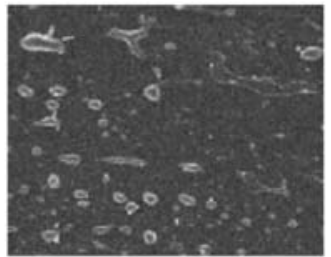

c) $\mathrm{PP}+20 \% \mathrm{PET}$

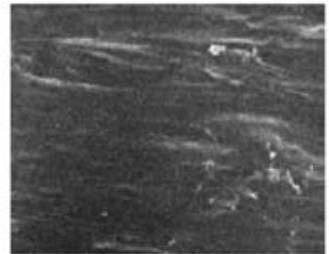

d) $\mathrm{PP}+30 \% \mathrm{PET}$

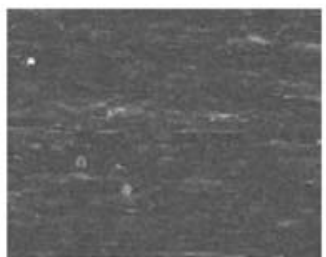

e) $\mathrm{PP}+40 \% \mathrm{PET}$

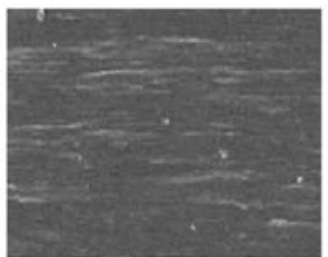

f) $\mathrm{PP}+50 \% \mathrm{PET}$

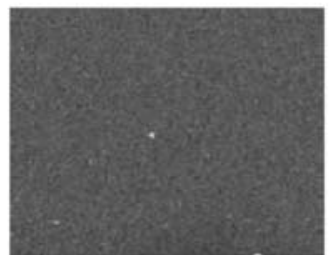

g) $\mathrm{PP}+60 \% \mathrm{PET}$

Figure 1.SEM micrographs of the PP/PET blends (X50)

In summary from the images obtained from Figure 1, it is observed that as the PET ratio increases, the increasing PET particles tend to merge and become fibrous, and as the addition ratio increases, these fibrils disappear to form a more homogenous structure.

In samples with an additive rate of over $40 \%$ PET, this homogeneity has fallen again. This decrease in particle size is due to a chemical reaction between the MA groups of PP-G-MA and the terminal functional group of $\mathrm{PET}$ in the $-\mathrm{OH}$ or $-\mathrm{COOH}$ group. The mechanical properties of the work we have done previously support SEM images [6]. 


\subsection{FT-IR analysis}

Compared with the SEM results and the FTIR results from spectroscopic measurements, the results were consistent with the results. As observed in the SEM results, the result in Fig. 2 is consistent with FTIR results for 40\% PET. The PP-MA compatibilizer used led to slipping of the peaks. Changes in the positions of the bands for PP $+40 \%$ PET samples were observed in the form of rise and fall in peak length compared to pure PET or pure PP. Peak intensities were observed to increase with increasing PET ratio due to the modification of PET by PET.

It was observed that the peak intensity of $C=0$ stretching in the range of $1720-1725 \mathrm{~cm}^{-1}$ for $\mathrm{PP}+40 \%$ PET was higher than that for PP $+50 \%$ PET sample. This result is supported both by results obtained from mechanical properties and by SEM results.
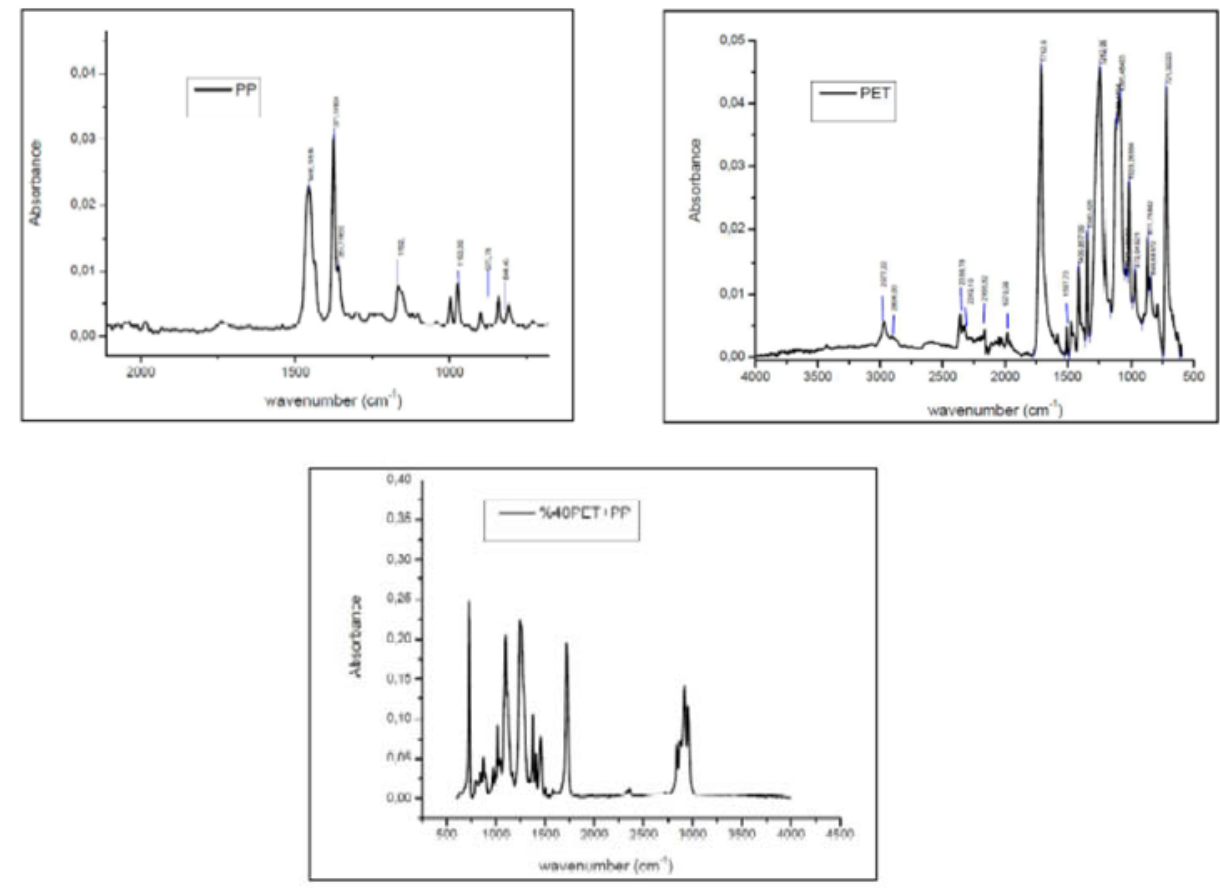

Figure 2.FT-IR Spectrum of the PP/PET blends (X50)

\section{Conclusions}

It has been investigated that the change in morphology and spectroscopic properties of the PP / PET blends with the PET additive is compatible. As the percentage of the mixture increases in the SEM images, there is a gap between the PET and the structure depending on the addition ratio. In the PP $+40 \%$ PET mixture, the transition from droplet form to fibril form appears clearly in the structure. This gives us knowledge of the healing of the works. In PP $+50 \%$ PET mixture, the homogeneity of the structure started to deteriorate again, the voids started to build up again and the structure became heterogeneous.

\section{References}

[1] Si X., "Preparation and study of polypropylene/polyethyleneterephthalate composite fibres.", Composites Science and Technology 68 (2008): 2943-2947 
[2] Torunoğlu Turan G., "Preparation and characterization of poly(ethyleneterephthalate) polypropylene blends"(PhD Diss., University of Istanbul Technical, 2012).

[3] Mirjalili, F., "Enhancing the Dyeability of Polypropylene Fibers by Melt Blending with Polyethylene Terephthalate.”, The Scientific World Journal, 2013.

[4] Çam, Ç., "Productıon Of Surface Coatıng Materıals From Post-Consumer Pet Bottles ", (M.Sc. Diss., University of Instanbul, 2015).

[5] Gartner, C., et al. "Grafting of Maleic Anhyride on Polyproplene and its effect on blending with poly(ethylene terephthalate." 48(2008):1910-1916.

[6] Kosovalı Çavuş F., "Modelling of Mechanical Properties of PP/PET Blends with Artificial Neural Networks" (paper presented at 9th International Physics Conference of the Balkan Physical Union - BPU9, Istanbul, Turkey, August 24 - 27, 2015). 